\title{
Valoración de las Competencias de Psicología: Estudio exploratorio en muestras de Estudiantes y Profesionales Activos
}

\section{Assessment of competences of Psychology: An exploratory study of samples of students and professionals}

Ximena Alejandra Suárez ${ }^{1}$

\section{Resumen}

Por medio de un cuestionario se caracterizan y comparan las percepciones de importancia y autovaloración en ocho competencias asociadas a la profesión de psicólogo, de 58 psicólogos activos de la Región de la Araucanía (Chile) y 46 estudiantes de los últimos cursos de la carrera de psicología de una Universidad Estatal de la misma región. Los resultados indican una mayor valoración para las competencias de diagnóstico y de intervención psicológica individual en ambos grupos; la existencia de diferencias significativas a favor del grupo de profesionales en la importancia asignada a las competencias evaluadas; y una autovaloración en los estudiantes significativamente menor al nivel de importancia que le asignan a las competencias en estudio.

Palabras clave: competencias, psicólogos, perfil profesional.

1 Psicóloga, Magíster en Educación. (C) Doctor en Psicología y Educación. Docente de la Carrera de Psicología de la Universidad Arturo Prat Sede Victoria. Chile. E-mail: ximena.suarez@unap.cl 


\begin{abstract}
The perception of valuation importance of eight competencies related to psychologist's profession, as well as the self-assessment in those competencies were characterized and compared. A sample of 58 psychologists from the Region of La Araucanía (Chile) and 46 students in the last courses of the career in a State University of the same Region was assessed. The results show a higher valuation of the competencies of diagnosis and psychological intervention in both groups. It was also found that the sample of professionals ascribe more importance to all competencies, as well as a lower valuation of competencies in self-assessment for the sample of students, when compared to the perception of the importance that they attribute to them..
\end{abstract}

Key words: skills, psychologist, professional profile. 


\section{Introducción}

Las instituciones de educación superior han debido asumir que en la formación de recursos humanos con la capacidad de responder a los requerimientos propios de la sociedad del conocimiento y la tecnología, el ajuste de las carreras a las necesidades de las sociedades, a nivel local y global, es un elemento de relevancia innegable (Beneitone, Esquetini \& González, 2007). Esto, a su vez, supone aceptar el desafío de concebir la organización educacional como sujeto de aprendizaje, capaz de reconocer, incorporar y adaptar la información que recibe del contexto para actuar de forma eficaz (Alles, 2005).

De acuerdo con Nagles (2005), una forma de generar respuestas efectivas a estos retos es preparar y ayudar a las personas para que desarrollen las competencias necesarias con el fin de asegurar un buen desempeño personal y profesional, cuestión que ubica la formación de alumnos de pregrado como tema de discusión, que se focaliza en las condiciones y características que estos deben evidenciar una vez egresados y titulados. En respuesta a esta controversia ha surgido la creciente preocupación por la "calidad" de las propuestas formativas. En esta línea, las universidades han ido evolucionando a través de diversas etapas en el proceso de búsqueda de propuestas que, sin homogenizar la diversidad de la realidad educativa nacional, avancen hacia un sistema más unificado que garantice la calidad (Pey, 2007).

Las políticas de educación aplicadas en Chile desde 1981 han configurado un sistema de educación superior cuya cobertura se ha incrementado de manera constante, permitiendo el acceso a grupos de la población que antes no participaban en este tipo de educación (Brunner, 2009; Espinoza \& González, 2008).

Al respecto, Brunner (2009) observa que el proceso de acceso a la educación superior evidencia la presencia de universidades altamente selectivas y otras menos selectivas. Esta situación estaría dando cuenta del tipo de estudiante que atiende cada universidad y podría hacer creer que las diferencias en las condiciones de entrada de los estudiantes significarían diferencias en la calidad de los procesos educativos a que son expuestos, 
las cuales, a su vez, se expresarían al egreso de la titulación. No obstante, Pascarella, et al. (2006, citado en Cabrera, Energici, Larraín \& Moretti, 2008), concluyen que estudiar en una institución selectiva no asegura que el estudiante participe de experiencias determinantes en su desarrollo cognitivo.

Lo anterior sugiere la importancia de avanzar en la evaluación y mejoramiento del valor agregado que entrega la formación de pregrado, por sobre las características de entrada de los estudiantes, práctica que, si bien evidencia esbozos de desarrollo, no está actualmente instalada en la Educación Superior Chilena.

En el avance hacia el aseguramiento de la calidad de la educación, la formación por competencias se ha posicionando como el eje de las reformas e innovaciones en el diseño curricular, debido a la opción realizada por el gobierno chileno el año 1997, cuando se definió una nueva política de desarrollo de la educación superior que, junto con crear el programa de Mejoramiento de la Calidad de la Educación Superior (MECESUP), favoreció el rediseño de currículos por competencias.

La formación de profesionales psicólogos en Chile se modificó drásticamente el año 1982, cuando la incorporación de entidades privadas al nivel de educación superior propició el aumento de instituciones que dictan esta carrera, que hasta entonces sólo era impartida por dos universidades.

De acuerdo con Toro \& Miranda (citados en Villegas, Marassi $\&$ Toro, 2003), Psicología es una de las carreras en la que mejor se puede apreciar el proceso de masificación de la educación superior chilena; de los dos programas de psicología existentes hasta 1981, en el año 2005 ya se tenía registro de 118 programas de psicología, con una matrícula anual de 25000 estudiantes y una cifra aproximada de 2000 alumnos titulados al año.

A pesar de este panorama, solo en la última década se comienzan a realizar cuestionamientos que dan origen a investigaciones científicas sobre el desarrollo y estado actual de los programas de Psicología en Chile. Los resultados de estos trabajos evidencian concordancia con las conclusiones de los estudios realizados a nivel internacional, que señalan la enorme e 
inorgánica proliferación de carreras, el sesgo clínico bajo el predominio del modelo médico con que se forman los psicólogos, la irregular calidad en los proyectos y equipos humanos, y la ausencia de pautas básicas comunes para los diseños curriculares (Di Doménico, 2002).

Lo descrito ha contribuido a que asociaciones gremiales, universidades y el Estado manifiesten su preocupación por la regulación de la enseñanza de la disciplina psicológica, tanto a nivel de pregrado como de postítulo, a fin de establecer estándares mínimos que aseguren la "calidad" de las propuestas formativas.

No obstante, el único referente formal acerca de lo que debería entregar la formación en psicología, es la declaración formulada por la Comisión Nacional de Acreditación de Pregrado (CNAP), en año 2002 y revisada en el año 2007 (CNA-CHILE), la cual establece un perfil genérico con el que deben cumplir todas las carreras de psicología que se imparten en Chile. En términos generales, en ella se señala que el psicólogo es un profesional generalista de formación científica, cuyas competencias son el diagnóstico, la evaluación y la intervención en procesos que involucren a personas, grupos y organizaciones, con capacidad de discernimiento ético y respeto por la naturaleza humana en el ejercicio de la profesión.

Aunque existen estudios que dan cuenta de la importancia y dominio percibido por estudiantes de universidades chilenas acerca de competencias genéricas, como es el caso del Proyecto Tuning Latinoamericano, son escasas las investigaciones relativas a competencias en estudiantes de psicología, así como la especificidad de las competencias que estos deberían poseer al momento de ingresar al campo laboral. Solo en el último tiempo, ya sea por intereses al interior de las diferentes universidades (Cabrera, Energici, Larraín \& Moretti 2008; Sanhueza, 2008) o bien, por grupos o redes que trabajan en conjunto para definir las competencias propias según especialidad (Castro, 2004; Julia, 2006) han surgido trabajos que intentan discutir y/o levantar competencias específicas para la formación en Psicología. Ante este panorama, cualquier intento por entregar antecedentes empíricos que se puedan sumar a los ya señalados y que contribuyan al debate de formación de psicólogos en Chile, es relevante. 
En la región de la Araucanía, la carrera de Psicología se imparte en cinco universidades, dos estatales y tres privadas, que en conjunto ofrecen una matrícula anual que se aproxima a las 320 vacantes, y al ańo 2010 congregaban a alrededor de 155 estudiantes en la etapa inmediatamente anterior al ingreso al campo laboral. Una de las instituciones estatales es la Universidad Arturo Prat-Sede Victoria, cuya carrera de Psicología se crea en el año 2004 y a la fecha ha titulado aproximadamente 65 profesionales orientados a las áreas de psicología clínica, educacional y laboral (Universidad Arturo Prat-Sede Victoria, 2011) de quienes no se tiene antecedentes específicos acerca de su situación laboral ni de sus apreciaciones sobre la calidad y pertinencia de su formación profesional, situación que podría ser muy similar a la de otras instituciones educativas.

Dado lo expuesto, resulta oportuno realizar un estudio tendiente a caracterizar y comparar las percepciones de competencias de la profesión de psicólogo, valoradas por el mercado, según la apreciación de psicólogos activos y la de estudiantes que finalizan su carrera. En términos específicos se pretende: identificar las competencias que según la valoración realizada por profesionales psicólogos activos conforman el perfil de la profesión del psicólogo; identificar las competencias que los alumnos de la carrera de Psicología consideran más importantes y representativas del perfil profesional del psicólogo; comparar la valoración que hacen de las competencias los alumnos y los psicólogos activos; explorar el grado de auto-evaluación de competencias específicas del perfil del psicólogo que realizan los alumnos de la carrera de Psicología; y comparar el grado de auto-autoevaluación de competencias con la valoración de importancia que hacen los alumnos, de las mismas.

Los antecedentes que se obtengan informarán respecto de si la formación recibida por los estudiantes que están próximos a egresar, concuerda con los requerimientos que demanda el ejercicio de la profesión y podrían ser utilizados en la institución de la muestra para reflexionar respecto de la pertinencia de los planes de estudios en que se basa la formación de sus estudiantes, con las demandas que el medio laboral plantea. 
Paralelamente, se pretende destacar la importancia de la autoobservación de las alternativas de formación universitaria existentes a través de la realización de investigaciones con la finalidad de enriquecer y ajustar los programas a las demandas de la sociedad actual. En este sentido, los resultados que se desprendan de este estudio podrían ser incorporados por otras universidades para potenciar la calidad de los programas de psicología.

\section{Antecedentes teóricos y empíricos}

La formación en competencias profesionales es uno de los objetivos esenciales de la universidad actual; sin embargo, es relevante destacar que el interés por ellas no surge en el contexto universitario sino en el mundo del trabajo en la década de 1870 y que, recién, a partir de la década de 1970, se les comienza a utilizar como criterios científicos explicativos de la eficiencia de las personas en el medio laboral (González, R. \& González, V., 2008).

La adopción del enfoque de competencias en la educación superior nace de la necesidad de responder más adecuadamente al cambio social y tecnológico, como también a la organización del trabajo para adaptarse al cambio (Corvalán \& Hawes, 2005).

Según el Informe Delors (1996) una competencia es una composición propia de cada individuo que combina la capacitación y calificación, adquirida para la formación técnica y profesional, como el comportamiento social, la aptitud para el trabajo en equipo, la iniciativa, el gusto por el riesgo, entre otros. Levy-Leboyer (1997), indica que este concepto da cuenta de un repertorio de comportamientos, que unas personas dominan mejor que otras, en el que se integran aptitudes, rasgos de personalidad y conocimientos.

Para el Proyecto Tuning Europa, una competencia es una combinación dinámica de atributos que describe los resultados del aprendizaje de un proceso educativo, o cómo los aprendices son capaces de actuar al final de un proceso educativo (González \& Wagenaar, 2003); mientras que para la Organización para la Cooperación y el Desarrollo 
Económico, representa la capacidad para responder exitosamente a una demanda, tarea o problema complejo, movilizando y combinando recursos personales (cognitivos y no cognitivos) y del entorno (OECD, 2005).

En el año 2000, Brunner planteaba que era necesario que Chile desarrollara estrategias que permitiesen conectar los procesos formativos y la actuación profesional, cuestión que, según Herrera, Respreto, Uribe y López (2009), si se hace desde una perspectiva de competencias, permite disponer de un medio para evaluar la calidad en las instituciones educativas.

Existe acuerdo en distinguir entre competencias específicas en un campo de estudio y competencias genéricas (comunes y transferibles). Mientras las competencias específicas corresponderían a conocimientos teóricos y procedimientos propios de cada profesión, las genéricas representarían atributos compartidos que pudieran generarse en cualquier titulación y que son consideradas importantes por ciertos grupos sociales.

En este marco, las competencias profesionales dan cuenta de la integración de conocimientos, destrezas y actitudes que permiten el desempeño profesional de calidad y, por lo tanto, constituyen el resultado de un proceso de aprendizaje que deberá garantizar que los alumnos sean capaces de integrar los conocimientos, destrezas, actitudes y responsabilidades que exigen los perfiles profesionales (Rodríguez, Hernández \& Díaz, 2007).

En lo relativo a la adquisición de las competencias previamente comprometidas en los programas de psicología, a nivel internacional se puede citar los estudios de Castro (2004) y Freixa (2005). El primero fue realizado en Argentinay entre sus conclusiones revela un limitado ajuste entre las competencias que requiere el psicólogo para un excelente desempeño en la profesión y las habilidades que deben tenerse. Señala además que, desde la perspectiva de los expertos participantes en el estudio, el perfil promedio registra carencias básicas en una gran cantidad de áreas desde el punto de vista del potencial usuario del sistema, hallazgo que resulta llamativo frente a la gran cantidad de estudiantes y graduados de Psicología en este país. 
Por su parte, el estudio de Freixa (2005), realizado por la Agencia Nacional de Evaluación de la Calidad y Acreditación Española (ANECA), concluye que el grupo estudiado valora como más importantes las competencias de evaluación psicológica y diagnóstico (de preferencia individual); las obligaciones deontológicas; la comunicación con los usuarios; la definición de metas y algunas competencias sobre intervención; concediendo menos importancia a algunas competencias de intervención más grupales y a las competencias relacionadas con la evaluación de la intervención. Los futuros egresados les conceden una importancia levemente menor a las competencias genéricas que a las específicas.

A nivel nacional, uno de los referentes sobre las habilidades y competencias que debería evidenciar un profesional psicólogo chileno es el planteamiento de la CNA. Según este organismo, el perfil de formación de este profesional debería contener habilidades y competencias relativas a: el diagnóstico, planificación y evaluación de diversos procesos y programas en el área de la psicología educacional, organizacional, clínica y otros campos de aplicación de la realidad social; la definición, aplicación y evaluación de estrategias de intervención para atender necesidades preventivas, terapéuticas y de desarrollo de la conducta de individuos, grupos y organizaciones, a partir de una base teórica sólida; y el diseño y a la realización de actividades de investigación científica en la disciplina. También plantea el desarrollo de capacidades tales como el pensamiento crítico, el auto-aprendizaje, la educación continua, la formación integral y el discernimiento ético, las que se consideran propias de una formación universitaria (CNA- CHILE, 2007).

Los primeros antecedentes respecto al análisis de la formación de psicólogos en Chile son los estudios de Villegas (1997), Toro y Villegas (1999) y Villegas, Marassi y Toro (2003). En ellos se señala que la formación académica de estos profesionales no tiene criterios para diferenciar el grado académico de Licenciado en Psicología del título profesional de Psicólogo; tampoco para diferenciar una formación de postgrado de una de post-título. Además, los autores indican la falta de consenso respecto de las sanciones con que se enfrentan los problemas conductuales, de 
salud mental y éticos que se manifiestan durante la carrera y la ausencia de señalamientos respecto a los recursos que requiere una formación de calidad.

Una línea que han seguido las investigaciones que intentan evidenciar el resultado de los programas de formación universitaria son los informes de la situación laboral de los titulados. Aplicado esto a los psicólogos titulados en Chile, Urrutia (2002) informa que la mayoría de estos profesionales ejerce en el área clínica (67\%), aunque de ellos sólo el $18 \%$ está acreditado como psicoterapeuta.

Estos antecedentes son concordantes con los resultados de un estudio realizado el año 2006, a solicitud del Colegio de Psicólogos de Chile, el cual, a partir de una muestra de 1798 participantes, concluye que la principal área de especialización es la psicología clínica (40,7\%), seguida del área laboral (19\%), educacional $(10,2 \%)$ y social comunitaria $(9,3 \%)$, (Linn, 2007).

Cabe destacar que ambos estudios coinciden en señalar el complejo panorama laboral vivenciado por los profesionales psicólogos hoy en día, fenómeno que los investigadores relacionan directamente con la masificación de la educación superior y con los procesos de globalización. Específicamente sobre las competencias del psicólogo, la investigación de Cabrera et al. (2008), revela que algunas de las competencias consideradas más importantes por los alumnos y docentes participantes del estudio, son la capacidad de escucha, el manejo del conocimiento de la disciplina, competencias de análisis y comprensión; mientras que las habilidades técnicas, como el uso de pruebas psicológicas, no son consideradas como muy importantes. El hecho que para la mayoría de los encuestados todas las competencias son importantes es interpretado por los investigadores como una representación difusa de las competencias más relevantes para un psicólogo, y por otro, como la exigencia que se hace al psicólogo del dominio de una serie de competencias que podría catalogarse como abrumador. 


\section{Diseño}

Se utilizó un diseño transversal, descriptivo, exploratorio.

\section{Participantes}

Acorde a los objetivos del estudio la muestra la conformaron dos grupos de sujetos seleccionados de acuerdo a criterios de accesibilidad y disponibilidad para participar en la investigación. El primero corresponde a 46 estudiantes que al momento de la investigación se encontraban cursando cuarto o quinto año de la carrera de Psicología de la Universidad Arturo Prat, Sede Victoria (71,74\% mujeres, 28,26\% varones), con una edad promedio de 23,16 años ( $D S=2,06)$. El segundo grupo lo integran 58 profesionales psicólogos titulados activos (79,31\% mujeres y 20,69\%, varones), cuya edad promedio es de 39,52 años ( $D S=2,79)$, con al menos tres años de inserción en el mercado laboral de la Región de la Araucanía; de ellos un 65,52\% presenta entre 3 y 10 años de experiencia laboral, un $20,69 \%$ entre 11 y 15 años, un 10,34\% entre 16 y 20 años y un 3,45\% más de 20 años de experiencia. La distribución por área de desempeño laboral de estos profesionales se presenta en la Tabla 1.

Tabla 1: Distribución de áreas de trabajo de los profesionales

\begin{tabular}{|l|c|c|}
\hline \multicolumn{3}{|c|}{ Área de trabajo de los profesionales } \\
\hline & Frecuencia & Porcentaje \\
\hline Clínica & 16 & 27,59 \\
\hline Educacional & 2 & 3,45 \\
\hline Laboral & 4 & 6,90 \\
\hline Docencia & 6 & 10,34 \\
\hline Otra & 2 & 3,45 \\
\hline
\end{tabular}




\begin{tabular}{|l|c|c|}
\hline Clínica - comunitaria & 10 & 17,24 \\
\hline Clínica - educacional & 4 & 6,90 \\
\hline Clínica - laboral & 4 & 6,90 \\
\hline Clínica - laboral - comunitaria & 2 & 3,45 \\
\hline Comunitaria - docencia - investigación & 2 & 3,45 \\
\hline Docencia - investigación & 2 & 3,45 \\
\hline Clínica - docencia & 2 & 3,45 \\
\hline Educacional - laboral & 2 & 3,45 \\
\hline
\end{tabular}

Para conformar la muestra de psicólogos el cuestionario se envió a 100 profesionales registrados en bases de datos de organizaciones e instituciones diversas de la Región de la Araucanía que presentaban una experiencia laboral mínima de tres ańos.

\section{Instrumentos}

Para la recogida de la información se utilizó el Cuestionario de Valoración de Competencias de la Profesión de Psicólogo, cuya construcción se realizó ad-hoc para esta investigación, en base a la información de organizaciones formales representativas de la profesión del psicólogo (Declaraciones del Colegio de Psicólogos de Chile; los estándares entregados por la Comisión Nacional de Acreditación de Pregrado (CNAP); Comisión Nacional de Acreditación de Psicólogos Clínicos de Chile; Estudio del Grado de Psicología en España (2005), más los resultados de investigaciones en perfiles competenciales realizadas en Chile). De éstas, la definición de la CNAP constituye el eje principal.

A partir de las fuentes señaladas se construyó un perfil de las competencias más representativas de la profesión, el cual fue sometido a la validación de tres jueces expertos (profesionales psicólogos con más 
de 10 ańos de experiencia profesional) para evaluar la pertinencia de las competencias incluidas en el perfil.

Seguidamente se construyeron los ítems de la versión preliminar del cuestionario, los que se sometieron a la valoración de los mismos tres jueces quienes los evaluaron en los aspectos de suficiencia, coherencia, relevancia y claridad. El proceso anterior determinó la conformación del instrumento definitivo: 44 ítems, con respuesta de tipo Likert, de cuatro graduaciones.

Un análisis factorial exploratorio, realizado en base a la aplicación del cuestionario a una muestra piloto de 35 sujetos, entre estudiantes y profesionales psicólogos, da cuenta que los ítems del cuestionario evalúan ocho competencias: Evaluación psicológica y psicodiagnóstico individual; Evaluación y diagnóstico grupal y organizacional; Intervención psicológica individual; Diseño e implementación de programas de intervención psicológica grupal y/o organizacional; Evaluación de programas de intervención psicológica en diferentes ámbitos; Diseño y realización de actividades de investigación científica en la disciplina; Competencias transversales específicas de la profesión; y Compromiso ético (Ver anexo $1)$.

\section{Procedimiento}

A los estudiantes se les aplicó el cuestionario en horario de clase. En una primera ocasión debieron señalar el nivel de importancia que asignaban a cada una de las competencias descritas en los ítems. Al cabo de una semana respondieron el cuestionario indicando la autovaloración de la adquisición de cada una de las competencias; para este efecto solo se procedió a cambiar la consigna.

A los profesionales psicólogos se les hizo llegar el cuestionario vía correo electrónico, correo postal o por entrega personal. En coherencia a las finalidades del estudio, que es conformar un perfil de competencias para la carrera de Psicología y obtener información que permita retroalimentar al programa de formación universitaria en cuestión, los profesionales solo respondieron evaluando la importancia de las competencias. 


\section{Resultados}

El análisis de la fiabilidad realizado a través del método de consistencia interna, arroja para el instrumento total un alpha de Cronbach 0,91 ; en tanto que para las escalas (competencias) los valores alpha oscilan entre 0,78 y 0,45 (Ver Tabla 2).

Tabla 2: Índices de confiabilidad del instrumento

\begin{tabular}{|l|c|}
\hline Competencias & $\begin{array}{c}\text { Nivel de Confiabilidad } \\
\text { (Alpha de Cronbach) }\end{array}$ \\
\hline $\begin{array}{l}\text { Evaluación psicológica y psicodiagnóstico } \\
\text { individual }\end{array}$ & 0,76 \\
\hline $\begin{array}{l}\text { Evaluación y diagnóstico grupal y } \\
\text { organizacional }\end{array}$ & 0,74 \\
\hline Intervención psicológica individual & 0,78 \\
\hline $\begin{array}{l}\text { Diseño e implementación de programas } \\
\text { de intervención psicológica grupal y/u } \\
\text { organizacional }\end{array}$ & 0,58 \\
\hline $\begin{array}{l}\text { Evaluación de programas de intervención } \\
\text { psicológica en diferentes ámbitos }\end{array}$ & 0,44 \\
\hline $\begin{array}{l}\text { Diseño y realización de actividades de } \\
\text { investigación científica en la disciplina }\end{array}$ & 0,52 \\
\hline $\begin{array}{l}\text { Competencias transversales específicas de la } \\
\text { profesión }\end{array}$ & 0,70 \\
\hline Compromiso ético & 0,57 \\
\hline Cuestionario total & 0,90 \\
\hline
\end{tabular}

Considerando que las competencias se evalúan con diferentes cantidades de ítems, se optó por calcular el puntaje total de cada competencia, para luego ponderarlo por el número de ítems respectivo, lo que posibilita, además, categorizar los puntajes de acuerdo a la escala de asignación de puntajes original. 
Los valores descriptivos para la importancia otorgada por estudiantes y profesionales a cada una de las ocho competencias se presentan en la Tabla 3. Al comparar dichos valores entre ambos grupos, mediante la prueba t de Student para grupos independientes, se obtienen diferencias significativas en: Diseño e implementación de programas de intervención psicológica grupal y/o organizacional $(t=5,09 ; \mathrm{p}<0,001)$; Evaluación de programas de intervención psicológica en diferentes ámbitos $(t=2,30$; $\mathrm{p}<0,05)$; Diseńo y realización de actividades de investigación científica en la disciplina $(t=2,07 ; \mathrm{p}<0,05)$; Competencias transversales específicas de la profesión ( $t=3,96 ; \mathrm{p}<0,001)$; $\mathrm{y}$ Compromiso ético $(\mathrm{t}=2,39 ; \mathrm{p}<0,05)$. En todas ellas, el puntaje promedio de importancia es mayor en el grupo de profesionales que en el grupo de estudiantes (Ver Tabla 4).

Tabla 3: Estadísticos descriptivos de la importancia de las competencias para alumnos y profesionales

\begin{tabular}{|l|c|c|c|c|}
\hline & \multicolumn{2}{|c|}{ Grupo Alumnos } & \multicolumn{2}{c|}{ Grupo Profesionales } \\
\hline Competencia & Media & Desv. típ & Media & Desv. típ \\
\hline $\begin{array}{l}\text { Evaluación psicológica } \\
\text { y diagnóstico } \\
\text { individual }\end{array}$ & 3,61 & 0,38 & 3,46 & 0,52 \\
\hline $\begin{array}{l}\text { Evaluación y } \\
\text { diagnóstico grupal y } \\
\text { organizacional }\end{array}$ & 3,14 & 0,578 & 3,086 & 0,49 \\
\hline $\begin{array}{l}\text { Intervención } \\
\text { psicológica individual }\end{array}$ & 3,39 & 0,448 & 3,47 & 0,40 \\
\hline $\begin{array}{l}\text { Diseño e } \\
\text { implementación } \\
\text { de programas } \\
\text { de intervención } \\
\text { psicológica grupal y/u } \\
\text { organizacional }\end{array}$ & 2,91 & 0,42 & 3,28 & 0,32 \\
\hline
\end{tabular}




\begin{tabular}{|l|c|c|c|c|}
\hline $\begin{array}{l}\text { Evaluación de } \\
\text { programas de } \\
\text { intervención } \\
\text { psicológica en } \\
\text { diferentes ámbitos }\end{array}$ & 3,29 & 0,32 & 3,47 & 0,45 \\
\hline $\begin{array}{l}\text { Diseño y realización } \\
\text { de actividades de } \\
\text { investigación científica } \\
\text { en la disciplina }\end{array}$ & 2,79 & 0,56 & 3,01 & 0,53 \\
\hline $\begin{array}{l}\text { Competencias } \\
\text { transversales } \\
\text { específicas de la } \\
\text { profesión }\end{array}$ & 3,47 & 0,29 & 3,68 & 0,27 \\
\hline Compromiso ético & 3,35 & 0,50 & 3,60 & 0,57 \\
\hline
\end{tabular}

Tabla 4: Valores prueba t para la igualdad de medias de la Importancia de las Competencias para Alumnos y Profesionales

\begin{tabular}{|l|c|c|c|}
\hline \multicolumn{1}{|c|}{ Competencia } & $\mathrm{t}$ & $\mathrm{gl}$ & Sig. (bilateral) \\
\hline $\begin{array}{l}\text { Promedio evaluación } \\
\text { psicológica y psicodiagnóstico } \\
\text { individual }\end{array}$ & 1,67 & 102 & 0,09 \\
\hline $\begin{array}{l}\text { Promedio evaluación } \\
\text { y diagnóstico grupal y } \\
\text { organizacional }\end{array}$ & 0,54 & 102 & 0,58 \\
\hline $\begin{array}{l}\text { Promedio intervención } \\
\text { psicológica individual }\end{array}$ & 0,86 & 102 & 0,39 \\
\hline $\begin{array}{l}\text { Promedio diseńo e } \\
\text { implementación de programas } \\
\text { de intervención psicológica } \\
\text { grupal y/u organizacional }\end{array}$ & 5,09 & 102 & 0,00 \\
\hline $\begin{array}{l}\text { Promedio evaluación de } \\
\text { programas de intervención } \\
\text { psicológica en diferentes } \\
\text { ámbitos }\end{array}$ & 2,30 & 102 & 0,02 \\
\hline
\end{tabular}




\begin{tabular}{|l|c|c|c|}
\hline $\begin{array}{l}\text { Promedio diseńo y realización } \\
\text { de actividades de investigación } \\
\text { científica en la disciplina }\end{array}$ & 2,07 & 102 & 0,04 \\
\hline $\begin{array}{l}\text { Promedio competencias } \\
\text { transversales específicas de la } \\
\text { profesión }\end{array}$ & 3,96 & 102 & 0,00 \\
\hline Promedio compromiso ético & 2,39 & 102 & 0,01 \\
\hline
\end{tabular}

En cuanto a la importancia de las competencias medidas versus el grado de realización o de adquisición de las mismas por parte de los estudiantes, la Tabla 5 permite apreciar que en todas ellas los puntajes promedio de realización son más bajos que los puntajes promedio de importancia asignada. Las mayores brechas se presentan en las competencias de Intervención psicológica individual y de Evaluación y diagnóstico grupal y organizacional; mientras que la competencia de Diseño y realización de actividades de investigación en la disciplina es la que presenta menor brecha.

Tabla 5: Comparación de puntajes promedios de Importancia y Autoevaluación de competencias de los estudiantes

\begin{tabular}{|l|c|c|c|c|c|}
\hline & \multicolumn{2}{|c|}{ Promedio de Puntaje } & \multicolumn{3}{c|}{$\begin{array}{c}\text { Prueba de muestras } \\
\text { relacionadas }\end{array}$} \\
\hline Competencia & Importancia & Autoevaluación & $\mathrm{t}$ & $\mathrm{gl}$ & $\begin{array}{c}\text { Sig. } \\
\text { (bilateral) }\end{array}$ \\
\hline $\begin{array}{l}\text { Evaluación } \\
\text { psicológica y } \\
\text { psicodiagnóstico } \\
\text { individual }\end{array}$ & 3,61 & 3,22 & 5,72 & 45 & 0,00 \\
\hline $\begin{array}{l}\text { Evaluación y } \\
\text { diagnóstico } \\
\text { grupal y } \\
\text { organizacional }\end{array}$ & 3,13 & 2,53 & 5,64 & 45 & 0,00 \\
\hline
\end{tabular}




\begin{tabular}{|l|l|l|l|l|l|}
\hline $\begin{array}{l}\text { Intervención } \\
\text { psicológica } \\
\text { individual }\end{array}$ & 3,39 & 2,63 & 10,97 & 45 & 0,00 \\
\hline $\begin{array}{l}\text { Diseño e } \\
\text { implementación } \\
\text { de programas } \\
\text { de intervención } \\
\text { psicológica } \\
\text { grupal y/u } \\
\text { organizacional }\end{array}$ & 2,90 & 2,47 & 6,23 & 45 & 0,00 \\
\hline $\begin{array}{l}\text { Evaluación de } \\
\text { programas de } \\
\text { intervención } \\
\text { psicológica } \\
\text { en diferentes } \\
\text { ámbitos }\end{array}$ & 3,29 & 2,80 & 6,05 & 45 & 0,00 \\
\hline $\begin{array}{l}\text { Diseño y } \\
\text { realización de } \\
\text { actividades de } \\
\text { investigación } \\
\text { científica en la } \\
\text { disciplina }\end{array}$ & 2,79 & 2,69 & 1,03 & 45 & 0,30 \\
\hline $\begin{array}{l}\text { Competencias } \\
\text { transversales } \\
\text { específicas de la } \\
\text { profesión }\end{array}$ & 3,46 & 3,29 & 3,64 & 45 & 0,00 \\
\hline $\begin{array}{l}\text { Compromiso } \\
\text { ético }\end{array}$ & 3,34 & 3,18 & 2,01 & 45 & 0,05 \\
\hline
\end{tabular}

La misma tabla da cuenta que, al comparar ambos puntajes (importancia y grado de realización), mediante la prueba t de Student para grupos relacionados, se manifiestan diferencias significativas en siete de las ocho competencias evaluadas, todas en desmedro de la autoevaluación del grado de realización o de adquisición de ellas: Evaluación psicológica y diagnóstico psicológico individual ( $t=5,72$; $\mathrm{p}<0,001)$; Evaluación y diagnóstico grupal $(t=5,64 ; \mathrm{p}<0,001)$; Intervención psicológica 
individual $(t=10,97 ; \mathrm{p}<0,001)$; Diseño e implementación de programas de intervención psicológica $(t=6,23 ; \mathrm{p}<0,001)$; Evaluación de programas de intervención psicológica en diferentes ámbitos $(t=6,05 ; \mathrm{p}<0,001)$; Competencias transversales específicas de la profesión $(t=3,64 ; \mathrm{p}<0,01)$ y Compromiso ético $(t=2,01 ; \mathrm{p}<0,05)$ (Ver Tabla 5).

\section{Discusión}

En una primera aproximación es posible señalar que la valoración de competencias que realizan profesionales psicólogos y estudiantes de psicología es diferente.

Para los profesionales todas las competencias evaluadas son importantes. Sin embargo, destacan como muy importantes las competencias transversales y las relacionadas con el compromiso ético. Lo anterior podría indicar que el desempeño profesional del psicólogo obliga a poner en juego competencias de carácter integral, propias de las ciencias humanas y dirigidas a la permanente autoevaluación de su quehacer, que van más allá de "especialización psicológica".

Pudiese ser que estas competencias sean facilitadas en su desarrollo por los programas de formación profesional, los cuales invitan a una constante revisión de los procesos de desarrollo personal y emergen ante los desafíos que plantea el ejercicio laboral en la diversidad. De cierta manera el resultado encontrado coincide con las ideas planteadas por Delors (1996) y Levy-Leboyer (1997) quienes señalan que para desempeñarse con efectividad en el campo laboral actual, no sólo son importantes los saberes y las competencias específicas, sino también, son necesarios las actitudes, valores y características personales.

$\mathrm{Si}$ bien, también para los alumnos las ocho competencias evaluadas son importantes, sólo la competencia evaluación psicológica y psicodiagnóstico es considerada muy importante, resultado que coincide con los hallazgos obtenidos por la investigación realizada por Freixa (2005). Al respecto se puede plantear que este resultado podría ser consecuencia de una representación social del quehacer del psicólogo, la cual lo vincula, primordialmente, al área clínica. También podría estar reflejando la 
valoración de esta competencia como una habilidad que les permitiría desempeñarse en cualquiera de las áreas de trabajo más frecuentes (clínica, educacional y laboral) y que, por lo mismo, es de vital importancia para el desempeño del futuro psicólogo. Otra explicación es suponer que esta valoración respondiese a una característica del programa de formación que, a través de las distintas asignaturas y de los docentes responsables de impartirlas, ofrece experiencias de aprendizaje que relevarían la importancia de ellas sobre otras.

Resulta interesante que la segunda competencia en importancia para los estudiantes sean las competencias transversales específicas de la profesión. Esto podría estar dando cuenta de una construcción que han ido realizando los estudiantes en el transcurso de su proceso formativo, respecto de la relevancia que tendría para el adecuado desempeño laboral, la formación integral.

Las diferencias que se observan en la importancia de las competencias evaluadas entre futuros psicólogos y psicólogos profesionales activos no es exclusiva para la muestra estudiada, ni tampoco para la realidad chilena. La investigación realizada en Argentina por Castro (2004) da cuenta de resultados muy similares a los de este estudio.

Los resultados orientan hacia una reflexión respecto al desarrollo de competencias que favorece el programa evaluado versus la realidad laboral local. Aparentemente el desempeño del quehacer laboral actual estaría involucrando aspectos de tipo psicosocial, que requieren competencias para la planificación, ejecución y evaluación de diversas actividades, especialmente de tipo grupal, de carácter preventivo y de promoción, que estarían siendo demandadas por políticas públicas que se ejecutan a través de instituciones y no a través del ejercicio libre de la profesión, el que tradicionalmente ha estado asociado al ámbito clínico curativo. El panorama laboral que muestran los profesionales otorga pleno sentido al seńalamiento de la CNAP, en lo referido a la caracterización de la formación generalista del profesional psicólogo.

Si las discrepancias observadas entre estudiantes y profesionales se asumen en términos absolutos, los primeros se encontrarían en una 
gran desventaja al momento de ingresar al mercado laboral, por cuanto evidenciarían un nivel de desarrollo, de algunas competencias, inferior al requerido por el mercado laboral, cuestión que podría incidir negativamente en la calidad de su desempeño profesional y les obligaría a buscar y acceder a instancias de perfeccionamiento que favorecieran tales competencias, cuando ellas debieron haber sido desarrolladas por la formación de pregrado.

$\mathrm{Al}$ respecto cabe insistir en el rol que desempeñan los responsables de impartir la formación de los estudiantes de la muestra, quienes a través de su accionar con los alumnos, el cual responde a opciones personales y profesionales, podrían estar promoviendo, directa o indirectamente, la importancia de algunas competencias sobre otras.

Al explorar la autoevaluación que realizan los alumnos sobre la percepción del grado de realización de las competencias evaluadas, se observan datos interesantes. A lo más los alumnos se autoevalúan como bastante competentes en la mayoría de las competencias evaluadas. Las competencias que presentan mayores puntajes son: Evaluación psicológica y diagnóstico individual; Competencias genéricas y Compromiso ético. Sin embargo, en la competencia Diseño e implementación de programas de intervención psicológica grupal y/u organizacional, los alumnos se autoevalúan solo como algo competentes.

Las diferencias significativas que se presentan entre el grado de importancia y la auto-evaluación acerca del desarrollo en siete de las ocho competencias evaluadas, dan cuenta de una importante brecha que puede traducirse en diferentes explicaciones o interpretaciones: sentimientos de incompetencia o de no dominio frente a competencias; inseguridad; no desarrollo de esta habilidad por parte del programa, presencia de sesgos personales, etc. Sin duda es un área que es necesario profundizar desde diferentes aproximaciones, con el fin de mejorar los procesos de formación. En la mayoría de las competencias los alumnos no perciben que hayan logrado adquirir un nivel de desarrollo de sus capacidades que les permita sentirse satisfechos para desempeñarse con efectividad, a pesar que en poco tiempo egresarán de su carrera profesional. 


\section{Conclusiones}

La valoración que hacen los alumnos de los últimos años de la Carrera de Psicología de la Universidad Arturo Prat de las competencias asociadas al profesional psicólogo, evidencia una tendencia hacia un perfil clínico. Sin embargo, el que los alumnos asignen importancia no sólo a competencias específicas o técnicas, sino también, a factores transversales indica que ellos han desarrollado conciencia acerca de la necesidad de alcanzar un desarrollo integral de sus capacidades, para desenvolverse en un mundo globalizado, estableciendo la necesidad de incorporar nuevas competencias a su repertorio.

El perfil competencial que poseen los profesionales psicólogos es generalista. Si bien cerca de un $60 \%$ de la muestra de profesionales se desempeña, en forma parcial o completa, en el área clínica, ellos otorgan importancia no sólo a competencias de esta especialidad, sino también a capacidades relacionadas con otras áreas, tales como el área comunitaria, el área educacional y el área de la salud. Destaca la importancia que asignan a competencias para trabajar con grupos y/u organizaciones, que tradicionalmente estuvieron relegadas a un segundo plano, evidenciando las nuevas necesidades y tendencias del campo laboral de la psicología introducidas por las políticas públicas y sociales de las últimas décadas.

Por consiguiente, se manifiesta como altamente necesario que las universidades mantengan una comunicación estrecha con la realidad local, regional y nacional, con el fin de estar actualizadas en relación a la evolución de las características y requerimientos del medio laboral. De esta manera, la formación profesional que ellas entreguen será más congruente con la realidad social (Alles, 2005; Beneitone et al., 2007).

La distancia que se manifiesta entre el grado de importancia que los alumnos le asignan a diferentes competencias y la autoevaluación que hacen del grado de realización de las mismas, indica que a pesar de estar en la fase de finalización de la carrera, ellos no se perciben como eficaces en el manejo de la mayoría de competencias asociadas a la profesión de psicólogo. Este resultado puede significar que la formación entregada por la Universidad no está contribuyendo al desarrollo de las competencias necesarias para desempeñarse en el ámbito laboral, en los niveles requeridos, pudiendo existir explicaciones diver- 
sas que es necesario interpretar con cautela. Entre estas, el procedimiento de evaluación usado (autoevaluación); las expectativas de los estudiantes hacia el programa de formación, el momento en que se realiza la evaluación. Sin duda, es recomendable que las instituciones educativas cuenten con procedimientos diversos y sistematizados, al realizar evaluación de competencias.

Se estima necesario continuar realizando investigaciones que permitan verificar con exactitud cuál es la necesidad de profesionales psicólogos para el mundo actual; tradicionalmente el área clínica ha constituido un sello diferenciador y de identidad de la profesión de psicólogo. No obstante, en la actualidad, a pesar de que el trabajo clínico continúa siendo el área a la que más se dedican los psicólogos y un área de alta demanda, existen otras áreas que se han fortalecido y que, si bien también requieren de competencias clínicas y de trabajo individual, necesitan del complemento de otras competencias de orientación más grupal. La interrogante a resolver no es determinar si un área de especialización de la psicología es más relevante que otra, sino cómo formar a un profesional psicólogo con un dominio idóneo de las competencias que el medio laboral demanda.

También se presenta como relevante la exploración de las razones por las cuales profesionales y estudiantes valorizan con menor intensidad la competencia referida a la investigación científica, sobre todo cuando se está inmerso en un mundo globalizado en que el conocimiento se renueva continuamente.

Aún cuando la información recogida es relevante, considerando la escasez de información que se evidenciaba en el tema al momento de efectuar el estudio, tanto a nivel nacional y por sobre todo regional, las características de la muestra, así como las del cuestionario utilizado, obligan a ponderar las conclusiones alcanzadas y plantean el desafío de continuar desarrollando investigaciones que favorezcan la construcción de instrumentos psicométricamente adecuados para la evaluación de competencias específicas de la profesión de psicólogo.

Lo anterior puede resultar en un insumo a considerar por parte de las asociaciones gremiales colegiadas para la definición de un perfil profesional del psicólogo en competencias, que pueda servir de guía para todos los programas de psicología y se avance en la regulación de la calidad de estos. 


\section{Referencias}

Alles, M. (2005). Desarrollo del talento bumano basado en competencias. Buenos Aires: Granica.

Beneitone, P., Esquetini, C. \& González, J. (2007). Reflexiones y perspectivas de la Educación Superior en América latina. Informe final-Proyecto Tuning-América Latina 2004-2007. Universidad de Deusto.

Brunner, J.J. (2000). Educación superior y desarrollo en el nuevo contexto latinoamericano. [versión electrónica]. Revista de la Educación Superior Chilena. Recuperado el 12 de septiembre del 2007. http://www. mecesup.cl/mecesup1/difusion/revista/revista1B.pdf

Brunner, J.J. (2009) Educación superior en Chile. Instituciones, mercados y politicas gubernamentales (1967-2007). Santiago: Ediciones Universidad Diego Portales.

Castro, A. (2004). Las Competencias Profesionales del Psicólogo y las necesidades de perfiles profesionales en los diferentes ámbitos nacionales. Revista Interdisciplinaria, 21(2), julio-diciembre, 117-152.

CNAP-Comisión Nacional de Acreditación de Pregrado (2002). Propuesta: Sistema Nacional de Aseguramiento de la Calidad de la Educación Superior, Documento de trabajo. Recuperado el 10 de enero de 2011 de http://www.cnap.cl/docu_trab/docu_trab_f.htm

CNA- Chile (2007). Comisión Nacional de Acreditación. Propuesta: Sistema Nacional de Aseguramiento de la Calidad de la Educación Superior, Documento de trabajo revisado el 7 de marzo del 2007.

Corvalán, O. \& Hawes, G. (2005). Aplicación del Enfoque de Competencias en la Construcción Curricular de la Universidad de Talca, (versión electrónica). Recuperado el 12 de diciembre de 2010 de http://www. mecesup.cl/difusion/destacado/2005

Delors, J. (1996). La educación encierra un tesoro. Madrid: SantillanaUnesco.

Di Doménico, C. (2002). Formación Psicológica Ayery Hoy en Latinoamérica. Conferencia dictada en la Universidad Nacional Andrés Bello de Chile. Inédito.

Espinoza, Ó. \& González, L. E. (2008) Equidad y accesibilidad a la educación superior (1990-2003). En J. J. Brunner y C. Peña (Eds.) Reforma a la educación superior en Chile (pp.143-172). Santiago: Ediciones Universidad Diego Portales.

Freixa, M. (2005). Libro Blanco de Título de Grado en Psicología. Agencia Nacional de Evaluación de la Calidad y Acreditación (ANECA), Universidad de Barcelona, España. Recuperado el 17 de enero del $2011 \mathrm{de}$ http://www.uctemuco.cl/docencia/seminario/presentaciones/rpey.pdf 
González, R. \& González, V. (2008). Competencias Genéricas y Formación Profesional: Un Análisis desde la Docencia Universitaria. Revista Iberoamericana de Educación, mayo-agosto, 047, 185-209.

González, J. \& Wagenaar, R. (2003): Tuning Educational Structures in Europe. Informe Final - Proyecto Piloto, Fase 1, Bilbao, Universidad de Deusto.

Herrera, A., Retrepo, M., Uribe, A. \& López, C. (2009). Competencias académicas y profesionales del psicólogo. Revista Diversitas Perspectivas en Psicología, 5(2), 241-254.

Juliá, M. T. (2006). Competencias profesionales del Psicólogo Educacional: Una tarea asociativa. Revista de Psicología, 15(2), 115-130.

Levy-Leboyer, C. (1997). Gestión de las Competencias. Barcelona: Gestión 2000.

Linn, H. (2007). La situación laboral del psicólogo en Chile. Ponencia para el VII Congreso Nacional de psicología, 9 y 10 de Noviembre, Santiago-Chile. Recuperado el 10 de septiembre del 2010 de www.psitec.cl

Nagles, G. N. (2005). El desarrollo de competencias. Revista EAN, 54, 99-137.

OECD (2005). The definition and selection of key competences: Executive summary, Paris, Francia: Autor.

Pascarella, E., Cruce, T., Umbach, P., Wolniak, G., Kuh, G., Carini, R., Hayek, J., Gonyea, R., et al. (2006) Institutional selectivity and good practices in undergraduate education: how strong is the link? En P. Cabrera, M. Energeci, A. Larraín A. R, Moretti. La formación en psicología desde una perspectiva de competencias. Una contribución para el mejoramiento de la formación universitaria en Chile. Rev. Calidad en la Educación, 33, diciembre, 183-221.

Pey, R. (2007). Seminario de Evaluación de Competencias: Tuning América Latina. Centro Nacional Tuning Chile.

Rodríguez, R., Hernández, N. \& Díaz, M. (2007). Cómo planificar asignaturas para el aprendizaje de competencias. Documentos ICE, Universidad de Oviedo.

Sanhueza, J. (2008) Modelo de Desarrollo de Competencias en la Formación del Psicólogo. Psicología Organizacional Humana, 1(1), 145-172.

Toro, J. \& Villegas, J.F. (1999). Psicología en Chile. En M. Alonso \& A. Eargly (Eds.) Psicología en las Américas. (pp. 129-153). Caracas. Sociedad Interamericana de Psicología.

Toro, J.P. \& Villegas, J. (2001). Problemas Centrales para la Formación académica y el entrenamiento profesional del psicólogo en las Américas, Volumen I. Sociedad Interamericana de Psicología (SIP). 
Toro, J. \& Miranda, G. (2003). Acreditación de Psicólogos y de Carreras de Psicología en Chile. En J. F. Villegas, P. Marassi \& J. Toro. Problemas Centrales para la Formación Académica y en Entrenamiento Profesional de los Psicólogos en las Américas, Vol. III. (pp. 105-133). Sociedad Interamericana de Psicología (SIP).

Universidad Arturo Prat-Sede Victoria, 2011. Informe Diagnóstico Sede Victoria Universidad Arturo Prat. Documento de trabajo istitucional no publicado.

Urrutia, C. (2002). La Psicología en Chile. Revista de Psicología: Consejo General de Colegios Oficiales de Psicólogos. Junio, 82, 71-74.

Villarroel, V. (2007). Reforma Curricular y Metodología en la Formación de Pregrado, Universidad del Desarrollo. Ponencia VII Congreso Nacional de Psicología, 9 y 10 de Noviembre, Santiago, Chile.

Villegas, J.F. (1997). Antecedentes, situación y perspectivas para el Desarrollo Institucional de la Psicología en Latinoamérica. $\sigma^{\circ}$ Congreso Nacional de Psicología. Santiago, Chile.

Villegas, J.F. (1999). La formación académica y el entrenamiento profesional del psicólogo en Latinoamérica. Conferencia inaugural. Congreso Latinoamericano de estudiantes de Psicología. Santiago, Chile.

Villegas, J.F. (2007). Formación de psicología en Chile, 60 años de historia. Ponencia, II Congreso de Psicología, Universidad de Santiago de Chile.

Villegas, J.F., Marassi, P. \& Toro, J. (2003). Problemas Centrales para la Formación académica y el entrenamiento profesional del psicólogo en las Americas, Volumen III. Sociedad Interamericana de Psicología (SIP).

Villegas, J.F., Redondo, J. \& Marassi, P. (2006). Responsabilidad Social en la Formación del Psicólogo: diversidad y calidad. Conferencia 1 Congreso de Psicología Convocado por Escuelas de Psicología del CUE. Psicología: Ciencia y Profesión. Talca, 19 al 21 de Octubre del 2006.

Fecha de recepción: 07 de enero de 2011.

Fecha de aceptación: 20 de junio de 2011. 


\section{ANEXO 1:}

\section{CUESTIONARIO DE COMPETENCIAS PROFESIONALES ESPECÍFICAS Y TRANSVERSALES DEL PSICOLOGO}

\section{Edad:}

Sexo: M ( ) F ( )

\section{Años de Experiencia Profesional.}

Marque el que corresponda. - 3 a 5 años:

- 6 a 10 años:

- 11 a 15 años:

- 16 a 20 años:

- Más de 21 años:

\section{Área de Especialización en que más trabaja actualmente}

- Clínica:

- Laboral:

- Educacional:

- Comunitaria:

- Docencia:

- Investigación:

- Otra:

\section{Estimado Colega:}

El presente instrumento está destinado a evaluar el grado de importancia que usted le asigna en la actualidad a las diferentes competencias específicas y transversales del Psicólogo que en él se presentan, con un fin de investigación y de retroalimentación para la formación profesional de futuros psicólogos. Por favor, solicito realizar su valoración en cada una de ellas otorgando valores de 1 a 4, en donde 1 corresponde a la mínima importancia (poco importante); 2, algo importante; 3, bastante importante y 4, a la máxima importancia (muy importante). Marque su respuesta con una x en el número seleccionado. 
1= Poco importante 2= Algo importante 3= Bastante importante 4= Muy importante.

\begin{tabular}{|c|c|c|c|c|}
\hline COMPETENCIAS & \multicolumn{3}{|c|}{ Importancia } \\
\hline $\begin{array}{c}\text { 1. Ser capaz de planificar y conducir una } \\
\text { entrevista psicológica. }\end{array}$ & 1 & 2 & 3 & 4 \\
\hline $\begin{array}{c}\text { 2. Saber analizar el contexto donde se } \\
\text { desarrollan las conductas individuales, } \\
\text { los procesos grupales y organizacionales. }\end{array}$ & 1 & 2 & 3 & 4 \\
\hline $\begin{array}{c}\text { 3. Ser capaz de definir los objetivos y } \\
\text { elaborar el plan de una intervención en } \\
\text { función del propósito de la misma. }\end{array}$ & 1 & 2 & 3 & 4 \\
\hline $\begin{array}{c}\text { 4. Pensar en forma creativa y desarrollar } \\
\text { nuevas ideas y conceptos. }\end{array}$ & 1 & 2 & 3 & 4 \\
\hline $\begin{array}{c}\text { 5. Diseñar y elaborar propuestas de } \\
\text { investigación científica. }\end{array}$ & 1 & 2 & 3 & 4 \\
\hline $\begin{array}{c}\text { 6. Planificar la evaluación de los programas } \\
\text { y de las intervenciones. }\end{array}$ & 1 & 2 & 3 & 4 \\
\hline $\begin{array}{c}\text { 7. Ser capaz de confeccionar informes y } \\
\text { artículos de investigación. }\end{array}$ & 1 & 2 & 3 & 4 \\
\hline $\begin{array}{c}\text { 8. Saber aplicar estrategias y métodos de } \\
\text { intervención psicológica directos } \\
\text { sobre los contextos de grupos y/o } \\
\text { de organizaciones: construcción de } \\
\text { escenarios saludables, productivos, } \\
\text { eficientes... }\end{array}$ & 1 & 2 & 3 & 4 \\
\hline $\begin{array}{c}\text { 9. Apreciar la diversidad y la } \\
\text { multiculturalidad. }\end{array}$ & 1 & 2 & 3 & 4 \\
\hline $\begin{array}{c}\text { 10. Selección y aplicación de baterías de test } \\
\text { acordes a las necesidades de la situación } \\
\text { de evaluación. }\end{array}$ & 1 & 2 & 3 & 4 \\
\hline $\begin{array}{c}\text { 11. Reconocer los propios sentimientos y } \\
\text { saber expresarlos adecuadamente. }\end{array}$ & 1 & 2 & 3 & 4 \\
\hline $\begin{array}{c}\text { 12. Implementar tareas de prevención de } \\
\text { riesgo y manejo de situaciones de crisis } \\
\text { y emergencias en grupos. }\end{array}$ & 1 & 2 & 3 & 4 \\
\hline $\begin{array}{c}\text { 13. Ser capaz de realizar intervenciones } \\
\text { psicológicas en niños y adolescentes. }\end{array}$ & 1 & 2 & 3 & 4 \\
\hline $\begin{array}{c}\text { 14. Saber interpretar los resultados de las } \\
\text { evaluaciones psicológicas. }\end{array}$ & 1 & 2 & 3 & 4 \\
\hline $\begin{array}{c}\text { 15. Realización de tareas de selección } \\
\text { y reclutamiento de personal en } \\
\text { organizaciones. }\end{array}$ & 1 \\
\hline $\begin{array}{c}\text { involucrar en la intervención a los } \\
\text { destinatarios. }\end{array}$ & 2 & 3 & 4 \\
\hline
\end{tabular}




\begin{tabular}{|c|c|c|c|c|}
\hline $\begin{array}{l}\text { 17. Comunicarse oralmente con facilidad } \\
\text { y claridad con personas de diversos } \\
\text { niveles culturales. }\end{array}$ & 1 & 2 & 3 & 4 \\
\hline $\begin{array}{l}\text { 18. Manejar técnicas de psicoterapia } \\
\text { adecuadas para el tratamiento de } \\
\text { cuadros clínicos adultos. }\end{array}$ & 1 & 2 & 3 & 4 \\
\hline $\begin{array}{l}\text { 19. Crear y poner en marcha programas } \\
\text { comunitarios de intervención y/o de } \\
\text { prevención. }\end{array}$ & 1 & 2 & 3 & 4 \\
\hline $\begin{array}{l}\text { 20. Saber trabajar en equipos y en grupos } \\
\text { interdisciplinarios. }\end{array}$ & 1 & 2 & 3 & 4 \\
\hline $\begin{array}{l}\text { 21. Saber describir y evaluar los procesos de } \\
\text { interacción, la dinámica y la estructura } \\
\text { organizacional e ínter organizacional. }\end{array}$ & 1 & 2 & 3 & 4 \\
\hline $\begin{array}{l}\text { 22. Redacción de informes psicológicos para } \\
\text { profesionales y no profesionales. }\end{array}$ & 1 & 2 & 3 & 4 \\
\hline $\begin{array}{l}\text { 23. Realización de intervenciones } \\
\text { psicológicas individuales en situaciones } \\
\text { de crisis y/o emergencias. }\end{array}$ & 1 & 2 & 3 & 4 \\
\hline $\begin{array}{l}\text { 24. Realizar evaluaciones y diagnósticos } \\
\text { clínicos siguiendo los criterios propios } \\
\text { de la profesión (modelos diversos, } \\
\text { criterios internacionales, DSM IV, CIE } \\
\text {-10). }\end{array}$ & 1 & 2 & 3 & 4 \\
\hline $\begin{array}{l}\text { 25. Conocer y ajustarse a las obligaciones } \\
\text { deontológicas de la Psicología. }\end{array}$ & 1 & 2 & 3 & 4 \\
\hline $\begin{array}{l}\text { 26. Elaboración de perfiles psicológicos en } \\
\text { diferentes ámbitos laborales a partir del } \\
\text { análisis de puestos y tareas. }\end{array}$ & 1 & 2 & 3 & 4 \\
\hline $\begin{array}{l}\text { 27. Exponer ante un público cuestiones y } \\
\text { temáticas psicológicas adaptándose a } \\
\text { sus contextos socioculturales. }\end{array}$ & 1 & 2 & 3 & 4 \\
\hline $\begin{array}{l}\text { 28. Desarrollar y mantener actualizadas } \\
\text { las propias competencias, destrezas y } \\
\text { conocimientos según estándares de la } \\
\text { profesión. }\end{array}$ & 1 & 2 & 3 & 4 \\
\hline $\begin{array}{l}\text { 29. Saber elegir las técnicas de intervención } \\
\text { psicológica adecuadas para alcanzar los } \\
\text { objetivos del tratamiento. }\end{array}$ & 1 & 2 & 3 & 4 \\
\hline $\begin{array}{l}\text { 30. Saber aplicar otras estrategias y métodos } \\
\text { de intervención sobre los destinatarios: } \\
\text { consejo psicológico, orientación } \\
\text { educacional y vocacional, mediación... }\end{array}$ & 1 & 2 & 3 & 4 \\
\hline $\begin{array}{l}\text { 31. Detección temprana de problemas de } \\
\text { aprendizaje en niños y adolescentes con } \\
\text { técnicas variadas. }\end{array}$ & 1 & 2 & 3 & 4 \\
\hline
\end{tabular}




\begin{tabular}{|c|c|c|c|c|}
\hline $\begin{array}{l}\text { 32. Diseñar, desarrollar y hacer seguimiento } \\
\text { de programas de capacitación de } \\
\text { personal en áreas diversas. }\end{array}$ & 1 & 2 & 3 & 4 \\
\hline $\begin{array}{l}\text { 33. Ser capaz de desarrollar intervenciones } \\
\text { psicopedagógicas en niños con } \\
\text { necesidades educativas especiales. }\end{array}$ & 1 & 2 & 3 & 4 \\
\hline $\begin{array}{l}\text { 34. Obtener información de forma efectiva } \\
\text { de diferentes fuentes: documentación } \\
\text { especializada, profesionales, otras } \\
\text { personas... }\end{array}$ & 1 & 2 & 3 & 4 \\
\hline $\begin{array}{l}\text { 35. Capacidad y disposición para intentar } \\
\text { la producción de nuevo conocimiento } \\
\text { mediante el manejo idóneo de } \\
\text { metodologías, técnicas y prácticas de } \\
\text { investigación. }\end{array}$ & 1 & 2 & 3 & 4 \\
\hline $\begin{array}{l}\text { 36. Saber describir y evaluar los procesos de } \\
\text { interacción, la dinámica de los grupos } \\
\text { y su estructura a través de variados } \\
\text { instrumentos. }\end{array}$ & 1 & 2 & 3 & 4 \\
\hline $\begin{array}{l}\text { 37. Aplicar programas de intervención } \\
\text { psicológica de grupos en áreas } \\
\text { educativas y/o comunitarias. }\end{array}$ & 1 & 2 & 3 & 4 \\
\hline $\begin{array}{l}\text { 38. Ser capaz de describir y medir variables } \\
\text { (personalidad, inteligencia y otras } \\
\text { aptitudes) y procesos cognitivos, } \\
\text { emocionales, psicobiológicos y } \\
\text { conductuales. }\end{array}$ & 1 & 2 & 3 & 4 \\
\hline $\begin{array}{l}\text { 39. Diseñar y aplicar programas y acciones } \\
\text { de prevención en el área de salud, } \\
\text { educación y comunidad. }\end{array}$ & 1 & 2 & 3 & 4 \\
\hline $\begin{array}{l}\text { 40. Ser capaz de medir y obtener datos } \\
\text { relevantes para la evaluación de } \\
\text { intervenciones. }\end{array}$ & 1 & 2 & 3 & 4 \\
\hline $\begin{array}{l}\text { 41. Valorar la actuación personal y conocer } \\
\text { las propias competencias y limitaciones. }\end{array}$ & 1 & 2 & 3 & 4 \\
\hline $\begin{array}{l}\text { 42. Ser capaz de seleccionar y construir } \\
\text { indicadores y técnicas de medición } \\
\text { para evaluar los programas y las } \\
\text { intervenciones. }\end{array}$ & 1 & 2 & 3 & 4 \\
\hline $\begin{array}{l}\text { 43. Mostrar interés por la calidad de la } \\
\text { propia actuación profesional y saber } \\
\text { desarrollar sistemas para garantizar la } \\
\text { calidad de los propios servicios. }\end{array}$ & 1 & 2 & 3 & 4 \\
\hline $\begin{array}{l}\text { 44. Conocer y aplicar las disposiciones } \\
\text { del código de ética del Colegio de } \\
\text { Psicólogos Chileno. }\end{array}$ & 1 & 2 & 3 & 4 \\
\hline $\begin{array}{l}\text { 45. Si existe una(s) competencia(s) que usted } \\
\text { considera como importante y no se } \\
\text { incorpora, por favor, agréguela... }\end{array}$ & & & & \\
\hline
\end{tabular}

